\title{
Rotator cuff troublemakers: pitfalls of MRI and ultrasound
}

This article was published in the following Dove Press journal:

Open Access Journal of Sports Medicine

24 December 2009

Number of times this article has been viewed

\author{
Christina M Chingkoe' \\ Jeremy H White ${ }^{2}$ \\ Luck J Louis ${ }^{2}$ \\ Gordon Andrews ${ }^{2}$ \\ Bruce B Forster ${ }^{2}$ \\ 'Faculty of Medicine, University \\ of British Columbia, Vancouver BC, \\ Canada; ${ }^{2}$ Department of Radiology, \\ University of British Columbia, \\ Vancouver BC, Canada
}

Correspondence: Bruce Forster Department of Radiology, University of British Columbia Hospital,

22II Wesbrook Mall, Vancouver BC, Canada V6T 2B5

$\mathrm{Tel}+\mathrm{I} 6048227976$

Fax + I 6048229701

Email bruce.forster@vch.ca

\begin{abstract}
Rotator cuff pathology is routinely evaluated in many imaging centers with both magnetic resonance imaging (MRI) and ultrasound. Despite good diagnostic accuracy using each of these modalities, certain limitations persist. In this pictorial essay, we describe five potential "troublemakers" of rotator cuff pathology which are recurrent themes in our busy shoulder referral center. The comparison of imaging findings on MRI and ultrasound are discussed. An awareness of these potential pitfalls will help improve radiologists' diagnostic accuracy of rotator cuff pathology, and allow the clinician to optimize imaging referral and better interpret the subsequent report.
\end{abstract}

Keywords: rotator cuff, ultrasound, MRI, correlation, shoulder

\section{Introduction}

Rotator cuff pathology is a common source of shoulder pain. Ultrasound (US) and magnetic resonance imaging (MRI) are two of the most widely used imaging tools to investigate such symptoms. Despite comparable diagnostic accuracy, limitations of each modality exist.

This pictorial essay illustrates five rotator cuff pathologies, or rotator cuff "troublemakers", where the diagnosis can be problematic. The advantages and shortcomings of both MRI and US in these specific examples will be outlined.

\section{Calcific tendinopathy}

Calcium deposition is easily seen on plain film as a density comparable to bone (Figure 1). Calcium deposits are also readily identified on US as lobular hyperechoic foci within the tendon, with strong acoustic shadowing in $79 \%$, faint acoustic shadowing in $14 \%$ and less commonly, no shadowing in $7 \%$ of rotator cuff calcifications. ${ }^{1}$ As calcium reflects the US beam, the shadows produced deep to the deposits increases their conspicuity (Figures $2 \mathrm{a}$ and $2 \mathrm{~b}$ ). MRI is not suitable for first line imaging of calcific tendinopathy, as calcium deposits appear as vague regions of low signal intensity, similar to that of adjacent tendons, and are generally not visible (Figures $2 \mathrm{c}, 2 \mathrm{~d}$ ). ${ }^{2}$ However, sometimes, the posterior acoustic shadowing caused by calcium deposition with US can be large enough to obscure an underlying rotator cuff tear (Figures 3a). If there is a strong clinical suspicion for a rotator cuff tear in a patient with calcific tendinopathy, MRI is recommended for further evaluation (Figures $3 \mathrm{~b}$ and $3 \mathrm{c}$ ). Another advantage of US is that it can be used for image-guided therapy, such as barbotage, in the treatment of calcific tendinopathy (Figures $4 \mathrm{a}$ and $4 \mathrm{~b}$ ). submit your manuscript $\mid$ www.dovepress.com

Dovepress
Open Access Journal of Sports Medicine 20 I0:I I-9

(C) 2010 Chingkoe et al, publisher and licensee Dove Medical Press Ltd. This is an Open Access article which permits unrestricted noncommercial use, provided the original work is properly cited. 


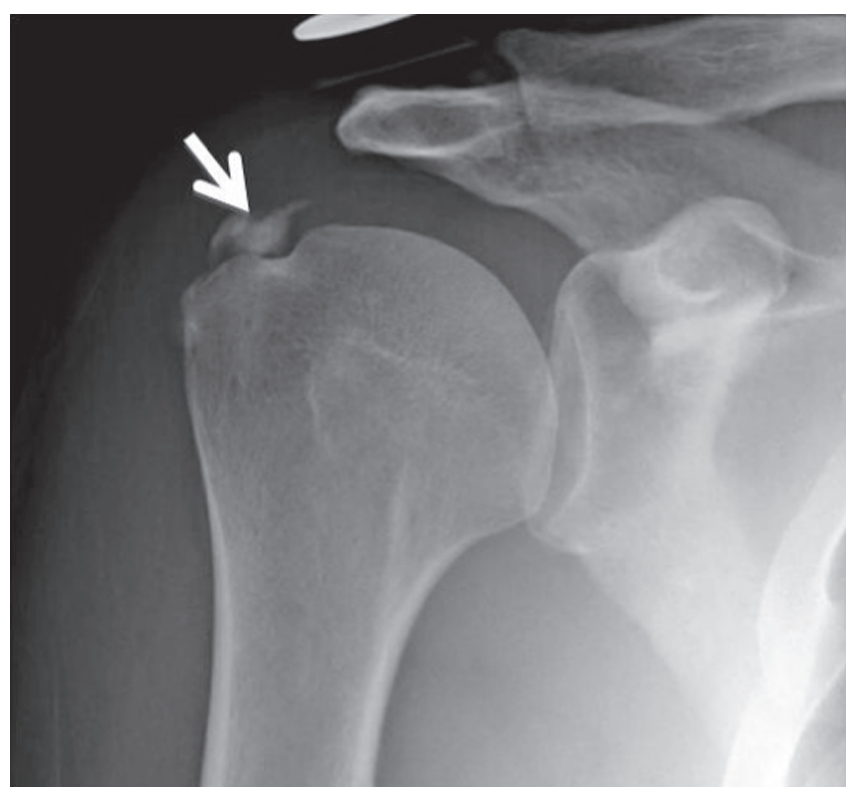

Figure I AP radiograph of the shoulder. Calcium deposition (arrow) is seen within the expected location of the supraspinatus tendon. Abbreviation: AP, antero posterior.

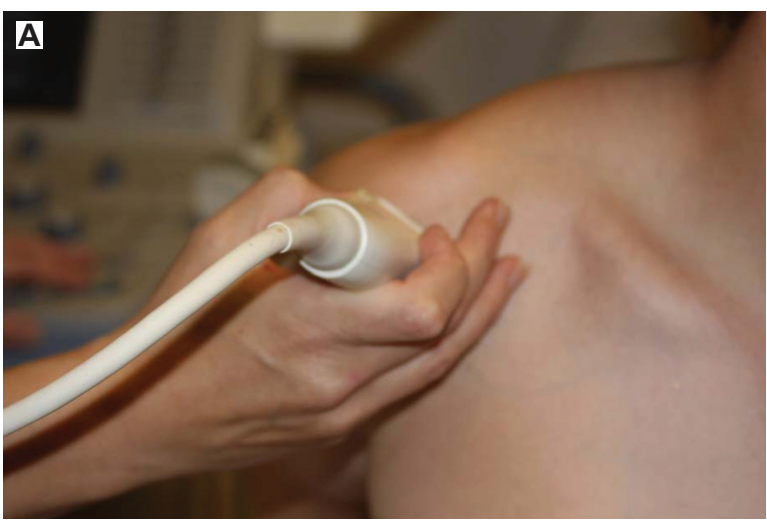

Figure 2a Ultrasound probe in short axis of the supraspinatus tendon.

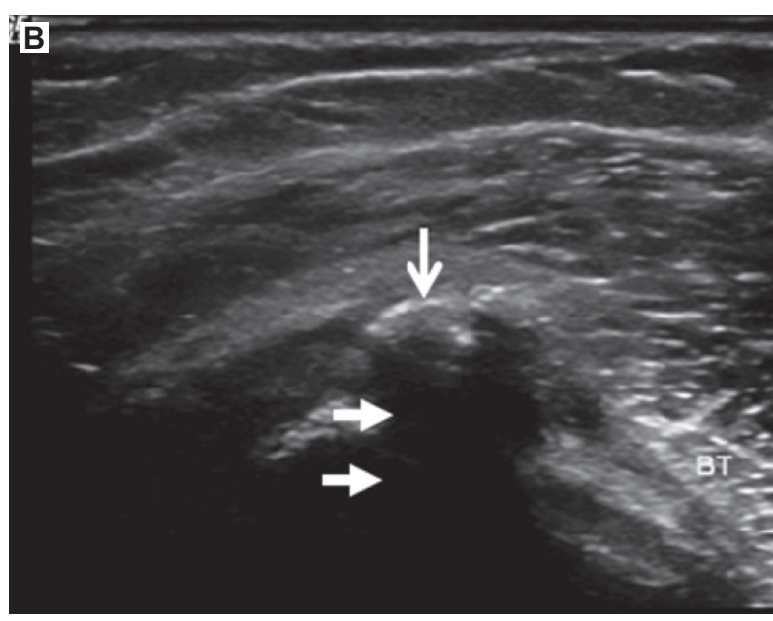

Figure $\mathbf{2 b}$ Short axis sonographic view of the supraspinatus tendon. Calcium deposition is seen as a hyperechoic focus (thin arrow) creating marked posterior acoustic shadowing (thick arrows).

Abbreviation: BT, biceps tendon.
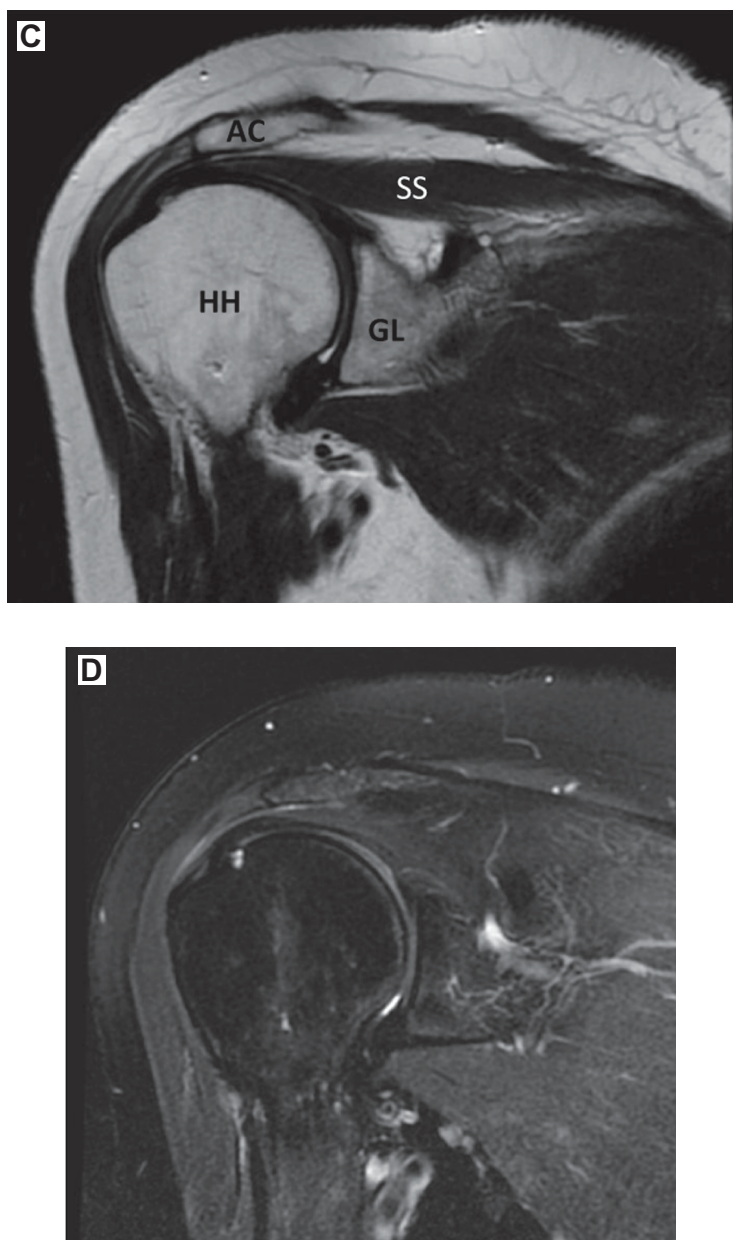

Figure 2c and d Coronal TI (c) and coronal T2 fat-saturated (FS) (d) MR images in the same patient as $2 \mathrm{~b}$. The calcium seen on the previous US is not appreciated. Abbreviations: $\mathrm{HH}$, humeral head; $\mathrm{AC}$, acromion; GL, glenoid; SS, supraspinatus; US, ultrasound. 


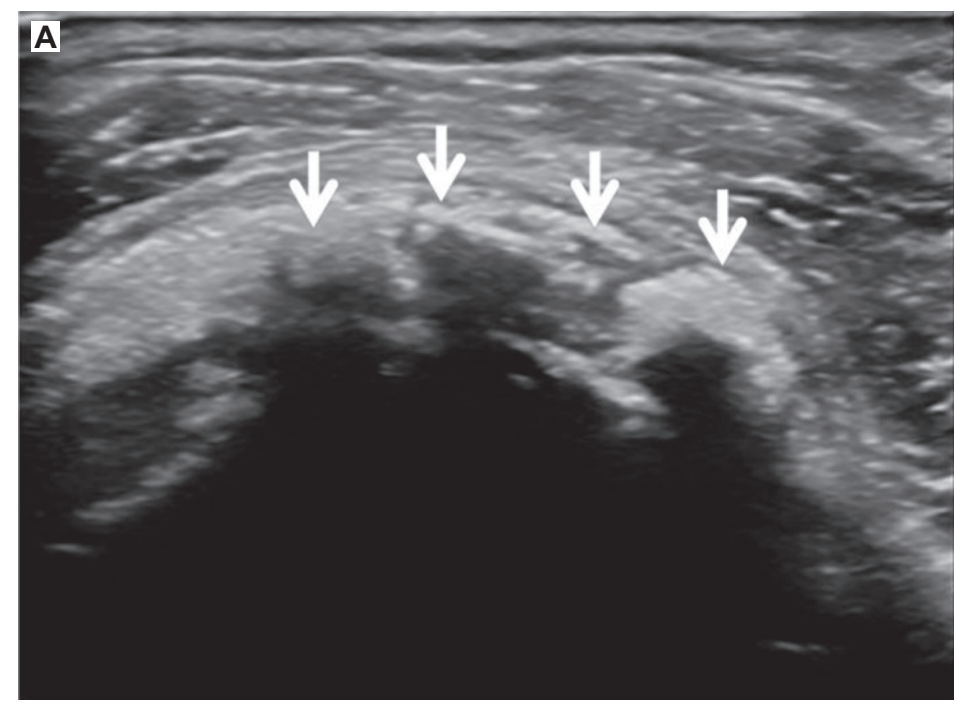

Figure 3a Short axis sonographic view of the supraspinatus tendon demonstrates extensive calcium deposition (arrows) with marked posterior acoustic shadowing. An underlying rotator cuff tear cannot be excluded in this case.
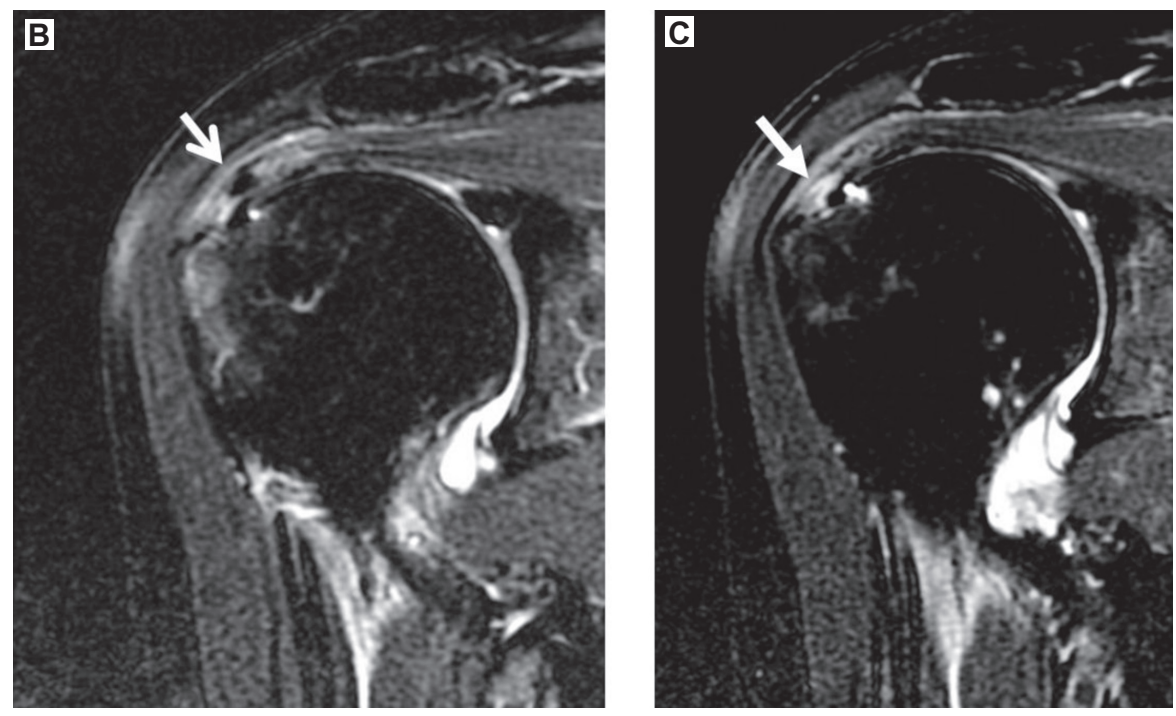

Figure $3 \mathbf{b}$ and $\mathbf{c}$ Coronal T2FS MR images in the same patient as 3a reveal a focus of calcification (b) adjacent to a partial thickness articular sided tear of supraspinatus (c). Abbreviation: $\mathrm{FS}$, fat saturated.

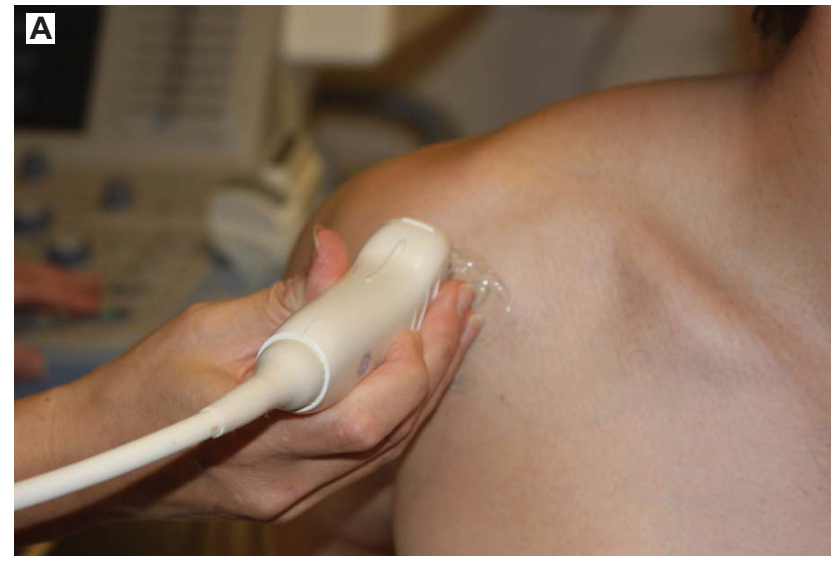

Figure $4 \mathrm{a}$ Ultrasound probe in long axis of the supraspinatus tendon.

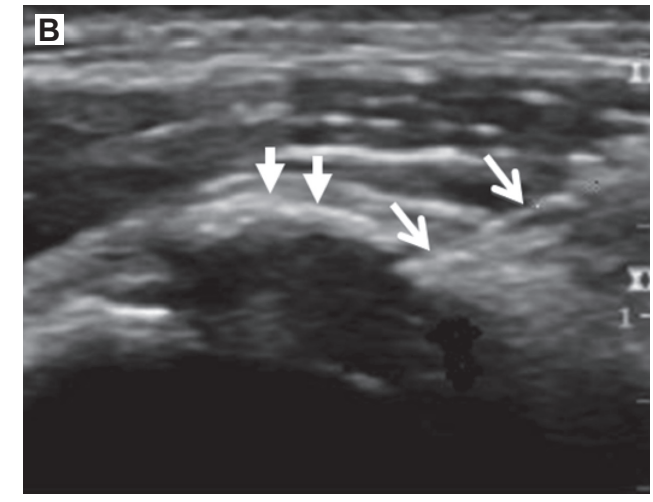

Figure 4b Long axis of US-guided barbotage of calcific tendinopathy. The needle tip (thin arrows) can be seen penetrating the calcium deposit (thick arrows). Abbreviation: US, ultrasound. 


\section{Teaching point}

US is the imaging modality of choice for diagnosing and potentially treating calcific tendinopathy. MRI can be useful in excluding an underlying rotator cuff tear in the setting of calcium deposition.

\section{Partial thickness tears}

Partial thickness tears are typically the most challenging rotator cuff pathology to diagnose, as they can be small and are not associated with tendon retraction. Ultrasound has excellent spatial resolution, which helps in the visualization of partial thickness tears; however, accurate sonographic evaluation requires proper technique, with the beam placed perpendicular to the tendon being examined. Even slight angulation of the beam can lead to hypoechoic defects which can be misinterpreted as tears. This artifact is known as anisotropy and is one of the most common causes for false-positive readings on US. ${ }^{3}$

With MRI, a partial thickness tear is diagnosed when T2 fluid signal intensity extends into either the bursal or the articular surface of the tendon, with the latter being more common. ${ }^{4}$ Intrasubstance extension of these tears can occur, as can isolated intrasubstance tears. Unfortunately, US and MRI do not always correlate with respect to diagnosing these tears (Figures 5a, 5b, 5c; 6a, 6b). With US, the sensitivity for detecting partial tears is variable, ranging from $36 \%$ to $93 \%$

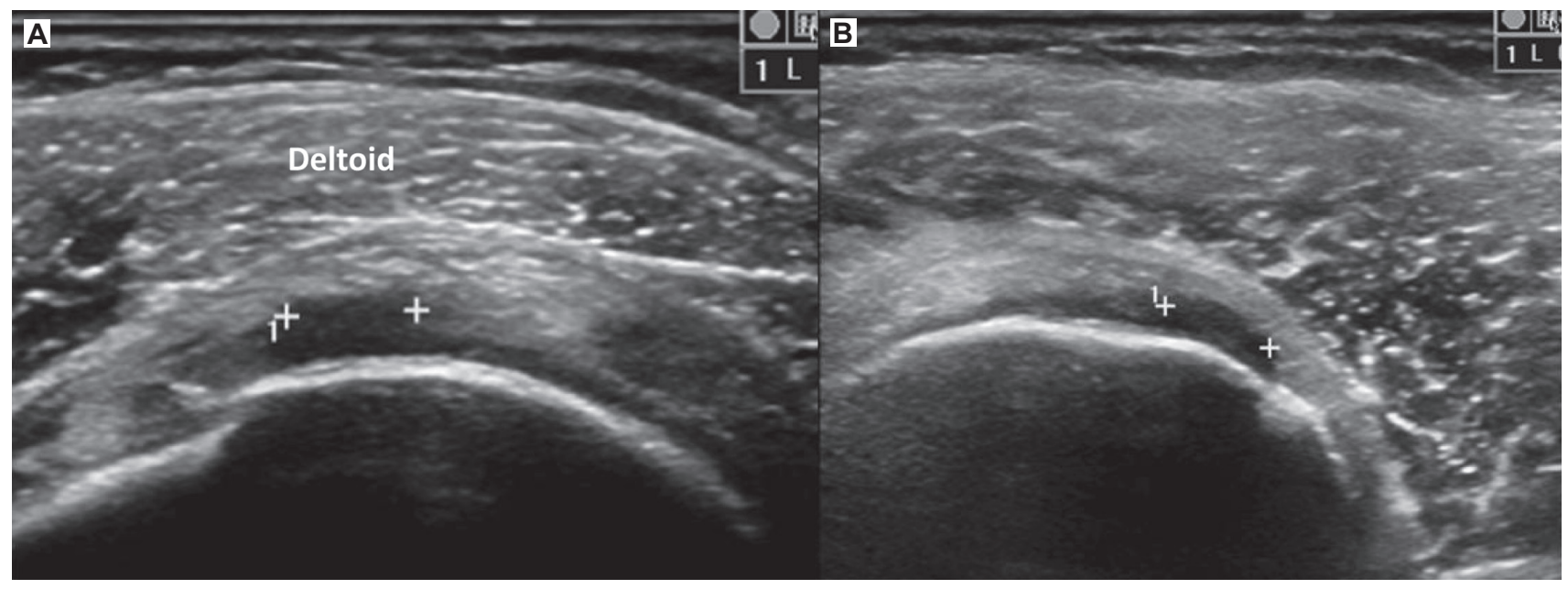

Figure $\mathbf{5} \mathbf{a}$ and $\mathbf{b}$ Short axis (left) and long axis (right) sonographic images of the supraspinatus tendon. A hypoechoic defect in the tendon is identified (calipers) suggesting a focal articular sided partial thickness tear.

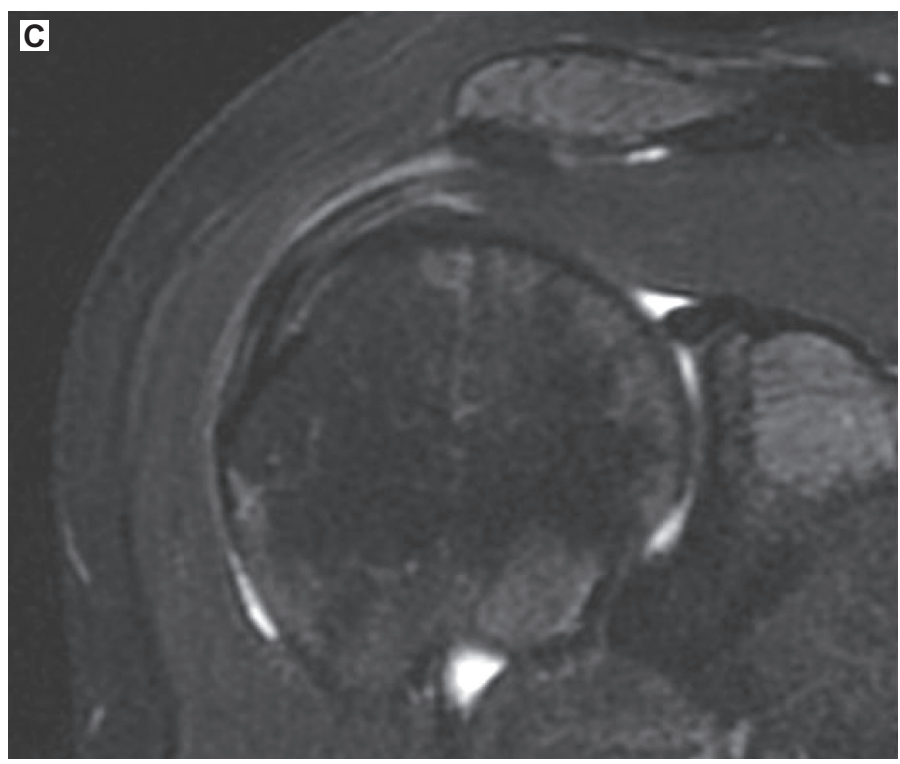

Figure 5c Coronal T2FS MR image in the same patient as 5a and b. Tendinopathy, but no tear, is identified. Abbreviation: FS, coronal fat saturated. 


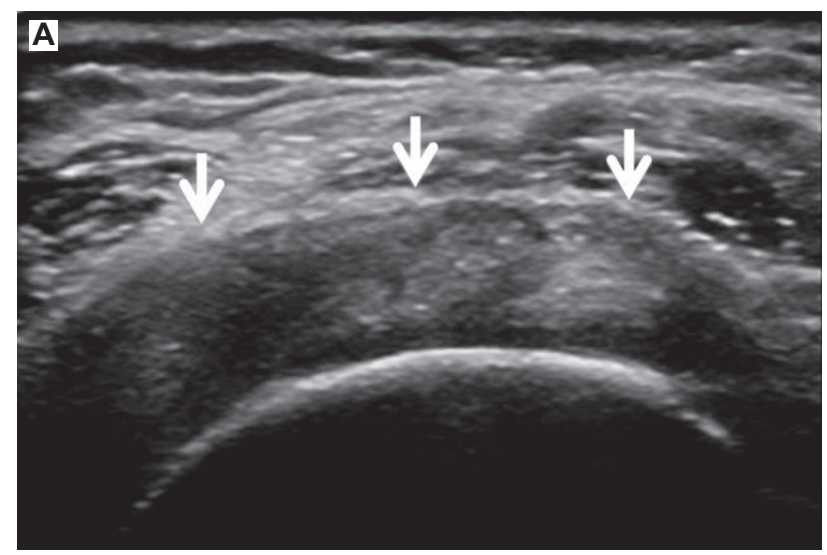

Figure 6a Short axis sonographic views of the rotator cuff demonstrating a thickened inhomogeneous supraspinatus tendon, compatible with tendinopathy (arrows).

and the specificity from $75 \%$ to $94 \% .{ }^{3}$ With conventional MRI, the sensitivity for detecting partial tears is relatively low, ranging from $35 \%$ to $50 \%$, while the specificity ranges from $85 \%$ to $97 \% .{ }^{5,6}$ In the older population, partial thickness tears are usually managed conservatively. In younger patients, partial thickness tears are increasingly seen as a surgically treatable source of pain. ${ }^{7}$ If it will affect patient management, MRI arthrography is recommended in the diagnosis of partial thickness tears as it has an overall accuracy of $95 \%$ for the most common articular-sided tears. ${ }^{8}$ One of the reasons why articular sided partial thickness tears may be better appreciated on MRI arthrography than conventional MRI is that a coapted tear is spread apart by the administration of intraarticular contrast under pressure. (Figures $7 \mathrm{a}, 7 \mathrm{~b}){ }^{9}$

\section{Teaching point}

US, based on its superior spatial resolution and being a dynamic examination, is the best non-invasive modality to

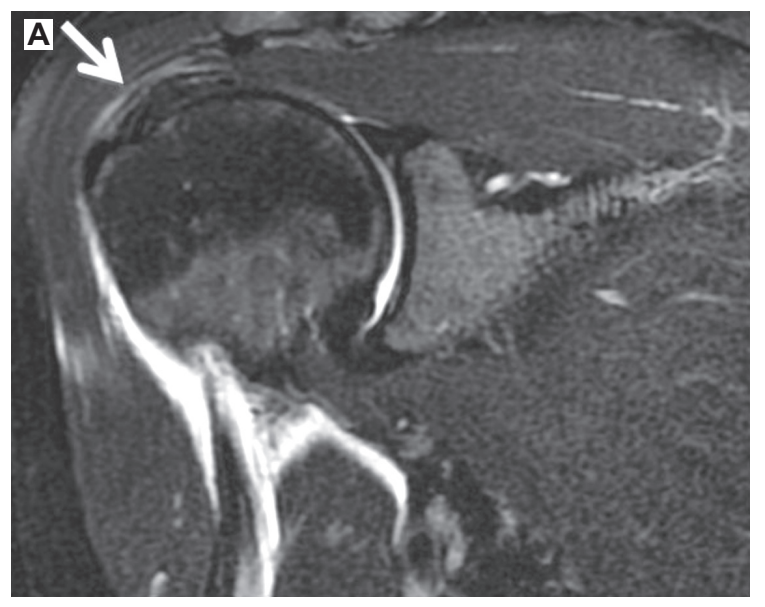

Figure 7a CoronalT2FS MR image. The supraspinatus tendon is mildly thickened with increased signal, compatible with tendinopathy. Otherwise, it appears intact. Abbreviation: $\mathrm{FS}$, fat saturated.

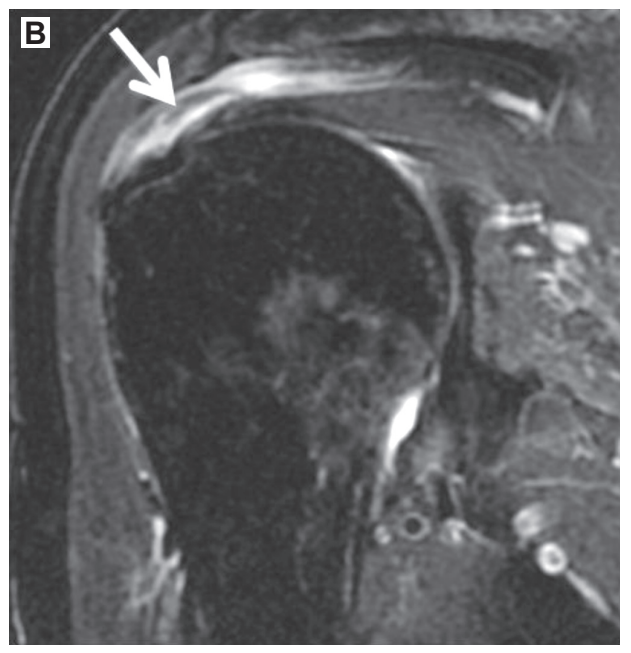

Figure $6 \mathbf{b}$ Coronal T2FS weighted MR images in the same patient as 6a demonstrates a high grade bursal sided and intrasubstance partial thickness tear (arrow). Abbreviations: FS, coronal fat saturated; MR, magnetic resonance.

generally assess for partial thickness tears. MRI arthrography is the most accurate imaging modality to evaluate partial thickness articular-sided tears.

\section{Full thickness tears}

Full-thickness tendon tears are generally easy to diagnose with both US and MRI, especially when there is associated tendon retraction. These tears are characterized by focal defects with fluid replacing the segment of torn tendon. However, certain conditions make the evaluation of full thickness tears more difficult to appreciate. For instance, full-thickness defects may occasionally be replaced by granulation tissues or

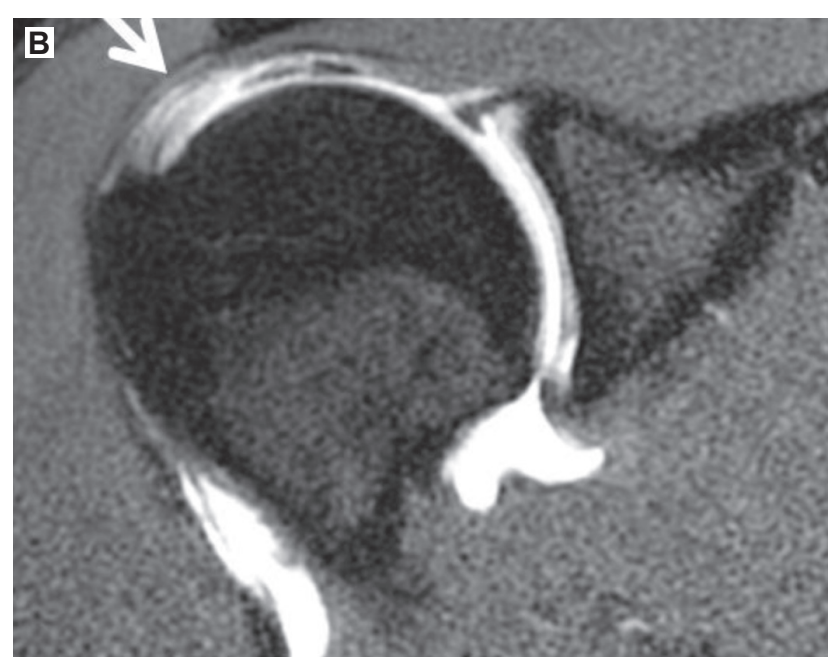

Figure 7b Coronal TIF5 MR arthrogram in the same patient as 7a, demonstrates a partial thickness articular sided and intrasubstance tear of the supraspinatus tendon. Abbreviations: FS, fat saturated; MR, magnetic resonance. 
hypertrophied synovium, rather than fluid. ${ }^{10}$ This can cause the tear to appear heterogeneous on US and be misinterpreted as tendinopathy (Figure 8a). If there is doubt regarding the diagnosis on US, MRI can help in making the distinction between tendinopathy and a tear (Figure 8b). In addition, the presence and degree of muscle atrophy and tendon retraction are optimally visualized on MRI (Figure 8c). Although fatty infiltration can be visualized on ultrasound as increased muscle echogenicity as well as a decreased pennate pattern, there is still no universal grading system to assess these secondary features of full thickness tears, and visualization of the retracted muscle under the acromion may be difficult. ${ }^{11}$ Tendon retraction and muscle atrophy are important to identify as they can affect suitability for surgical repair.

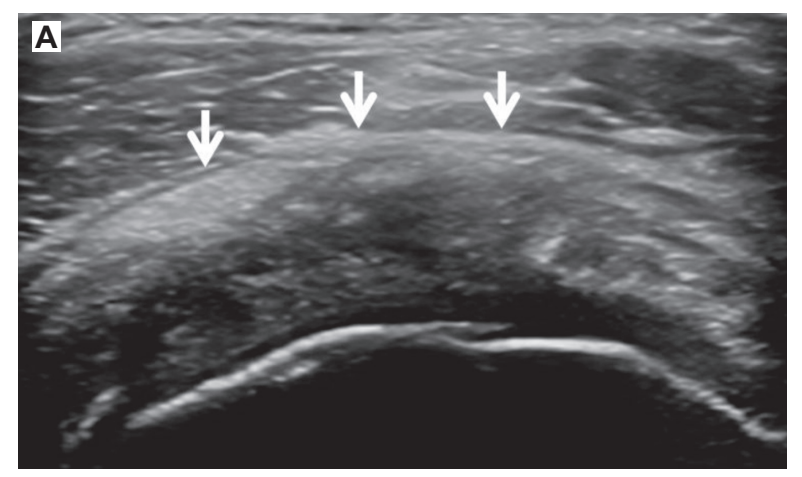

Figure 8a Short axis sonographic image of the supraspinatus tendon reveals a markedly heterogeneous tendon, suggesting tendinopathy (arrows). A full-thickness tear could not be excluded based on the heterogeneity and an MRI was performed. Abbreviation: MRI, magnetic resonance imaging.

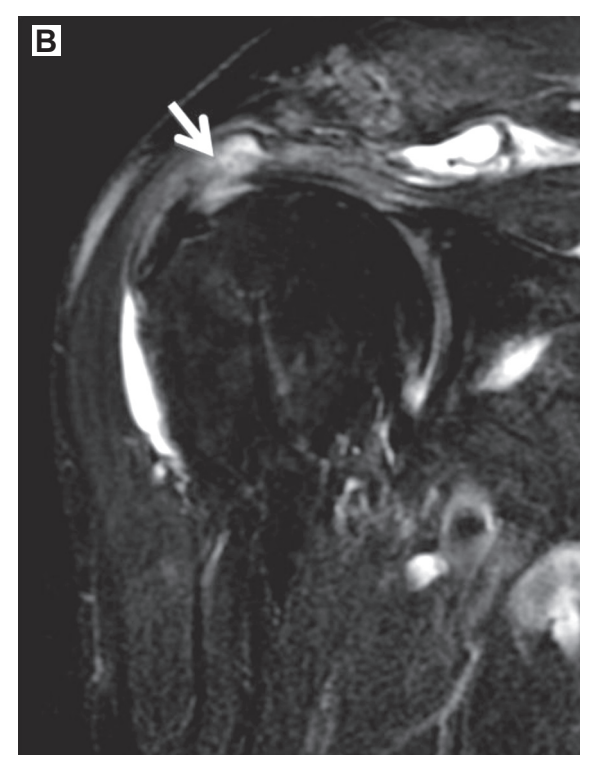

Figure $\mathbf{8 b}$ Coronal T2FS weighted MR images in the same patient as $8 \mathrm{a}$. There is a full-thickness tear (arrow) of supraspinatus. Granulation tissue may have masked the appearance of the tear on US.

Abbreviation: FS, fat saturated.

\section{Teaching point}

Full thickness tears can sometimes be obscured by granulation tissue making them difficult to appreciate on US. The degree of tendon retraction and the assessment of muscle volume loss are best assessed by MRI.

\section{Subscapularis tears}

Subscapularis is often considered the most difficult rotator cuff tendon to evaluate on both US and MRI. With US, a patient with subscapularis pathology may have limited external rotation which can make exposure of the tendon, and therefore identification of a tear, difficult. ${ }^{12}$ However, with careful examination tears can be demonstrated on US (Figure 9). With MRI, it is the location of the tear itself, rather than patient

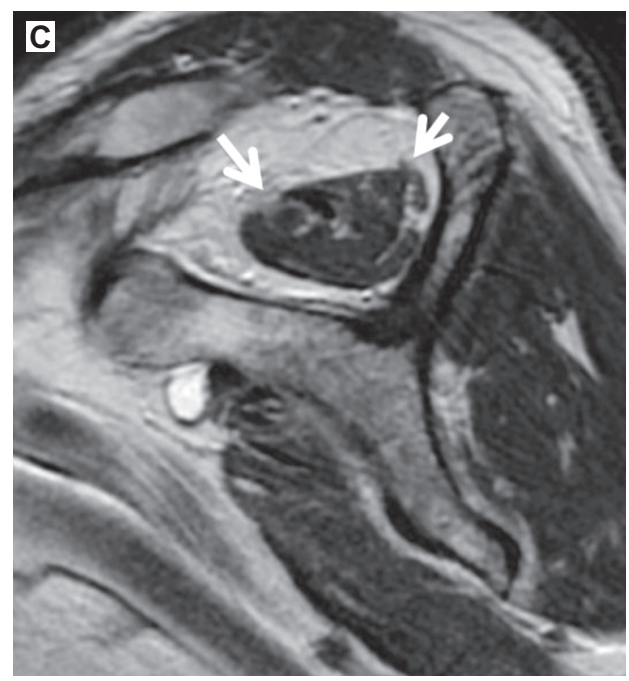

Figure 8c Sagittal T2 weighted MR image in the same patient as 8a and b. Moderate supraspinatus volume loss is noted (arrows).

Abbreviation: MR, magnetic resonance.

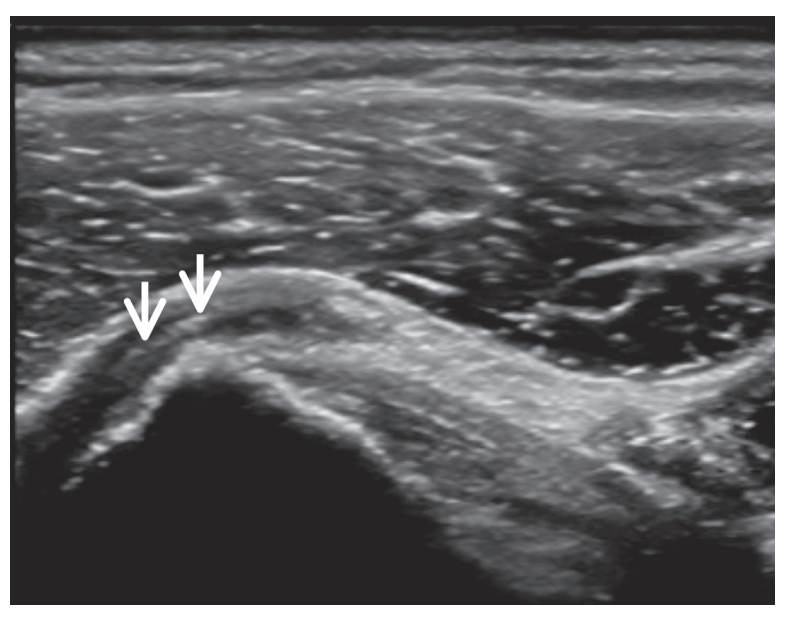

Figure 9 Short axis US image demonstrating an intrasubstance tear of subscapularis (arrows).

Abbreviation: US, ultrasound. 
positioning, which can cause them to be overlooked. Tears of the subscapularis usually begin on the articular surface of the superior fibers, near the insertion into the lesser tuberosity. These superior fiber tears may be missed because the inferior portion of the tendon appears normal. ${ }^{13}$ Our experience has shown that relying on the axial images alone is insensitive for these tears. The incorporation of fat saturation on the sagittal oblique T2 images improves the diagnostic accuracy for subscapularis tears (Figures 10a, 10b). ${ }^{14}$

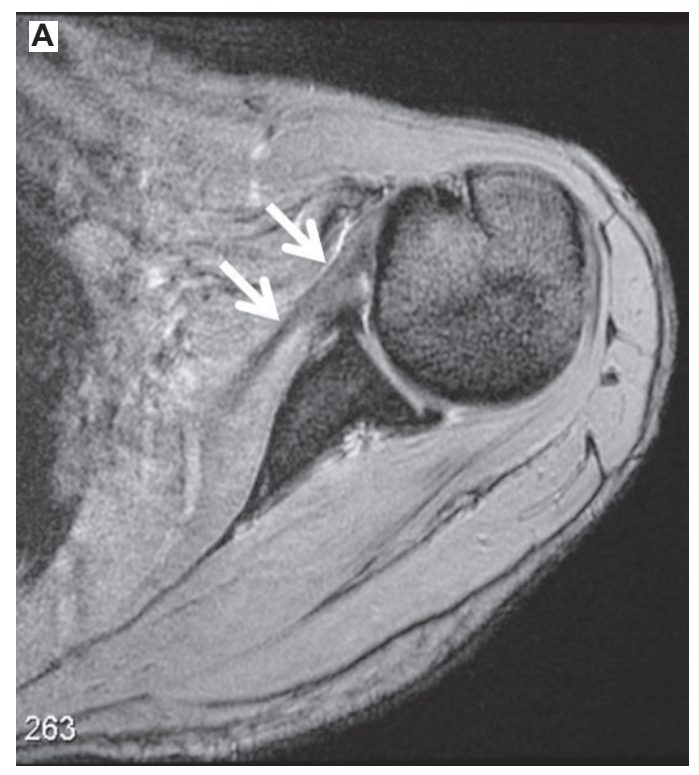

Figure I0a Axial GRE demonstrates an intact subscapularis tendon (arrows). Abbreviation: GRE, gradient echo.

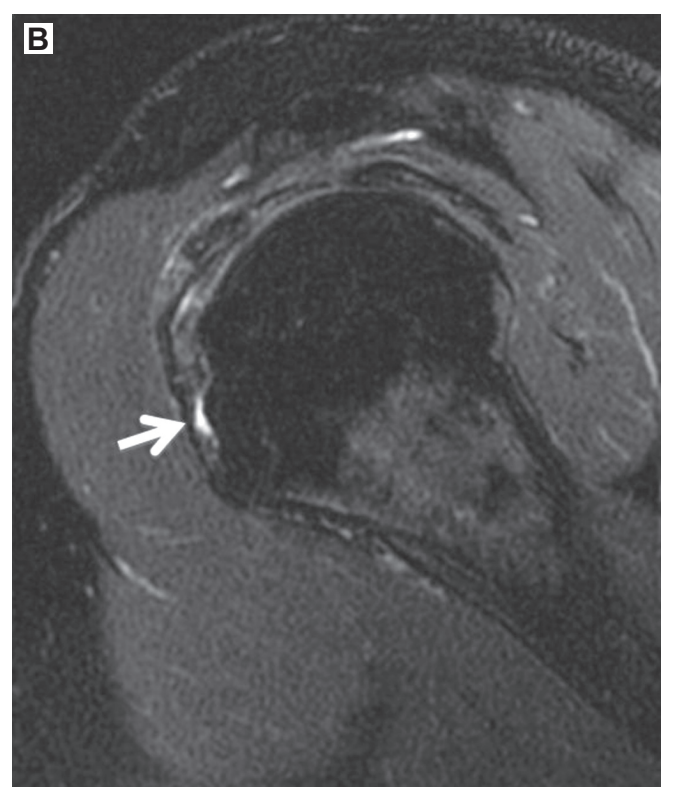

Figure I Ob Sagittal oblique T2FS MR image in the same patient as I0a demonstrates a partial thickness articular sided tear of the superior fibers of subscapularis (arrow). Abbreviations: FS, fat saturated; MR, magnetic resonance.
Sensitivity for subscapularis tears can also be improved by looking for secondary signs associated with subscapularis pathology, such as cysts in the lesser tuberosity (Figure 11). ${ }^{12}$ Full-thickness and intrasubstance tears of subscapularis are easily identified with either MRI or US, if there is associated medial dislocation of the long head of biceps tendon (Figure 11).

\section{Teaching point}

Subscapularis tears can be challenging to visualize on US because of limited patient positioning. With MRI, subscapularis tears are best visualized on oblique sagittal fat-suppressed sequences.

\section{Biceps tendinopathy}

The request to "exclude biceps tendinopathy" often accompanies the standard requisition for rotator cuff evaluation on MRI. Although full and split thickness tears can be accurately diagnosed on MRI, biceps tendinopathy is exquisitely illustrated on ultrasound. Ultrasound confers the benefit of a dynamic exam with excellent spatial resolution and the availability of color Doppler. Findings include tendon thickening, increased tendon echogenicity, excessive fluid within the sheath, and increased peritendinous vascularity (Figures 12a, 12b).

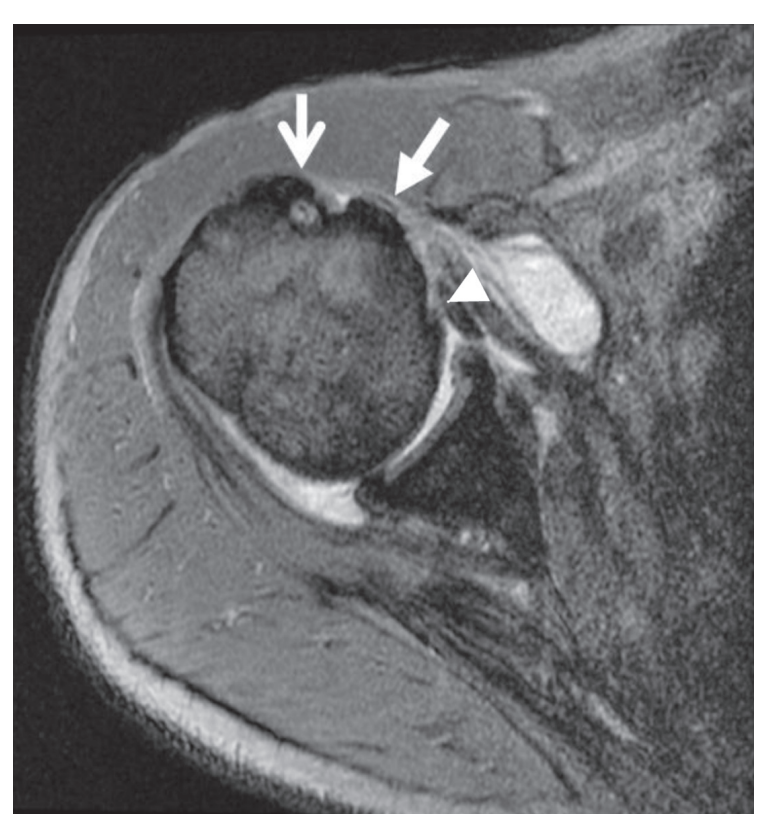

Figure I I Axial GRE image demonstrating the presence of a cyst (thin arrow) near the expected subscapularis insertion, in association with a full thickness tear (thick arrow) of the subscapularis tendon. Note the medial dislocation of the long head of biceps tendon (arrowhead).

Abbreviation: GRE, gradient echo. 


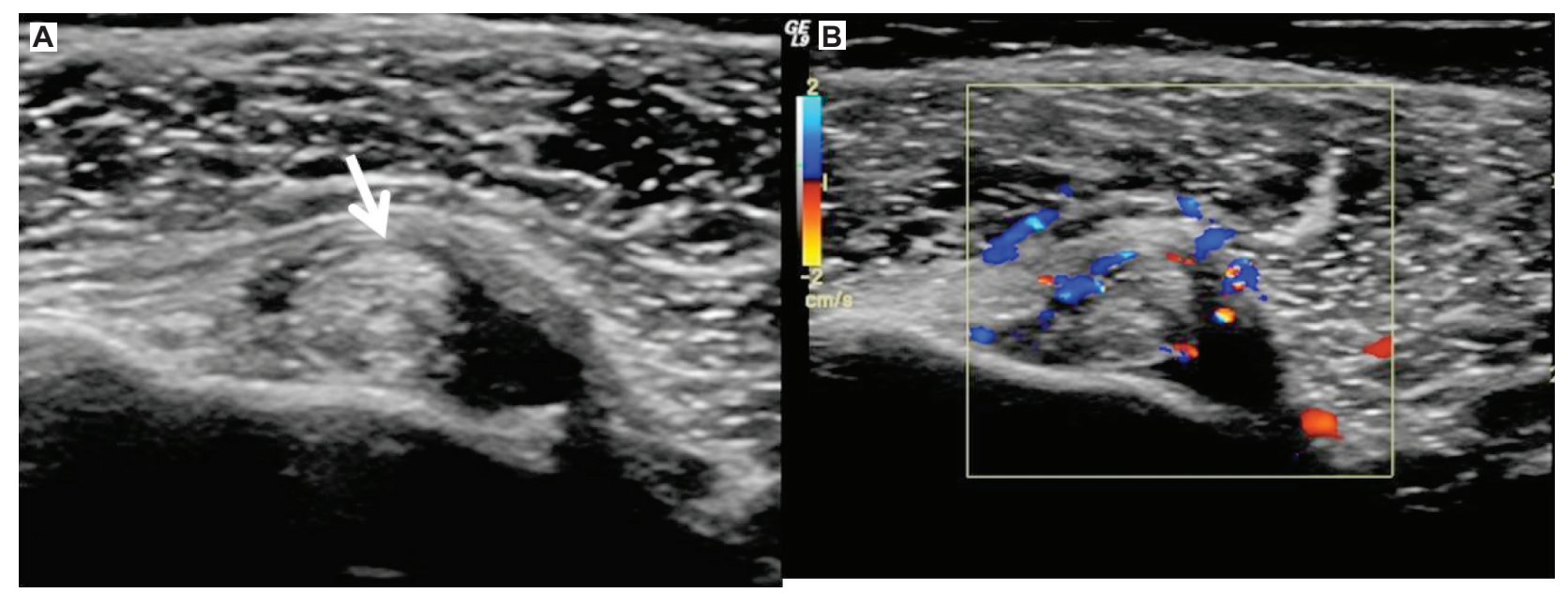

Figure I $2 \mathbf{a}$ and $\mathbf{b}$ Short axis ultrasound images without (left) and with color Doppler (right). The long head of biceps tendon is enlarged and heterogeneous with increased flow, compatible with tendinopathy. Note the small amount of fluid in the biceps tendon sheath (arrowhead).

The potential pitfall of MRI in the diagnosis of biceps tendinopathy is the "magic angle" effect. ${ }^{15}$ This is created when structures that have uniformly arranged collagen, like the biceps tendon, yield falsely hyperintense signals, mimicking tendinopathy or a tear, on MRI when they are imaged at an angle of 55 degrees to the main magnetic field. This artifact is seen with short transposable element (TE) sequences ( $\mathrm{TE}<37 \mathrm{~ms}$ ), such as $\mathrm{T} 1$, proton density, and gradient echo sequences. Therefore any signal change on a short TE sequence needs to be corroborated by signal change on a $\mathrm{T} 2$ weighted sequence (long TE), where the magic angle does not occur.

It should also be remembered that simply seeing fluid in the long head of biceps tendon sheath on MRI does not constitute tendinopathy. The tendon sheath normally communicates with the glenohumeral joint. However, if synovitis or tendon thickening/signal change is seen in the tendon sheath, biceps tendinopathy is likely.

\section{Teaching point}

Ultrasound with color Doppler exquisitely evaluates biceps tendinopathy. On MRI, false positives may occur due to the magic angle effect, so correlation with $\mathrm{T} 2$ sequences is needed. Ancillary findings like synovitis and signal changes within the tendon may indicate underlying tendinopathy.

\section{Conclusion}

US and MRI each have their advantages in the evaluation of rotator cuff pathology. Knowledge of the aforementioned pitfalls will allow the radiologist to understand the limitations of each test and to understand when to suggest further imaging in relation to the clinical question, as well as allow the clinician to optimize their imaging referral.

\section{Disclosures}

The authors has no conflicts of interest.

\section{References}

1. Farin PU, Jaroma H. Sonographic findings of rotator cuff calcifications. J Ultrasound in Medicine. 1995;14:7-14.

2. Zubler C, Mengiardi B, Schmid MR, Hodler J, Jost B, Pfirrmann CW. MR arthrography in calcific tendinitis of the shoulder: Diagnostic performance and pitfalls. Eur Radiol. 2007;17:1603-1610.

3. Rutten MJ, Jager GJ, Blickman JG. From the RSNA refresher courses: US of the rotator cuff: Pitfalls, limitations, and artifacts. Radiographics. 2006;26:589-604.

4. Tuite MJ, Turnbull JR, Orwin JF. Anterior versus posterior, and rimrent rotator cuff tears: Prevalence and MR sensitivity. Skeletal Radiol. 1998;27:237-243.

5. Balich SM, Sheley RC, Brown TR, Sauser DD, Quinn SF. MR imaging of the rotator cuff tendon: Interobserver agreement and analysis of interpretive errors. Radiology. 1997;204:191-194.

6. Martin-Hervas C, Romero J, Navas-Acien A, Reboiras JJ, Munuera L. Ultrasonographic and magnetic resonance images of rotator cuff lesions compared with arthroscopy or open surgery findings. J Shoulder Elbow Surg. 2001;10:410-415.

7. Meister K, Thesing J, Montgomery WJ, Indelicato PA, Walczak S, Fontenot W. MR arthrography of partial thickness tears of the undersurface of the rotator cuff: An arthroscopic correlation. Skeletal Radiol. 2004;33:136-141.

8. Waldt S, Bruegel M, Mueller D, et al. Rotator cuff tears: Assessment with MR arthrography in 275 patients with arthroscopic correlation. Eur Radiol. 2007;17:491-498.

9. Magee T, Williams D, Mani N. Shoulder MR arthrography: Which patient group benefits most? Am J Roentgenol. 2004;183:969-974.

10. Alasaarela E, Takalo R, Tervonen O, Hakala M, Suramo I. Sonography and MRI in the evaluation of painful arthritic shoulder. Br J Rheumatol. 1997;36:996-1000.

11. Khoury V, Cardinal E, Brassard P. Atrophy and fatty infiltration of the supraspinatus muscle: Sonography versus MRI. Am J Roentgenol. 2008;190:1105-1111. 
12. Teefey SA, Rubin DA, Middleton WD, Hildebolt CF, Leibold RA, Yamaguchi K. Detection and quantification of rotator cuff tears. comparison of ultrasonographic, magnetic resonance imaging, and arthroscopic findings in seventy-one consecutive cases. J Bone Joint Surg Am. 2004;86-A:708-716.

13. Tung GA, Yoo DC, Levine SM, Brody JM, Green A. Subscapularis tendon tear: Primary and associated signs on MRI. J Comput Assist Tomogr. 2001;25:417-424.
14. Studler U, Pfirrmann CW, Jost B, Rousson V, Hodler J, Zanetti M. Abnormalities of the lesser tuberosity on radiography and MRI: Association with subscapularis tendon lesions. Am J Roentgenol. 2008;191:100-106.

15. Peh WC, Chan JH. The magic angle phenomenon in tendons: Effect of varying the MR echo time. Br J Radiol. 1998;71:31-36.

16. Robinson P. Sonography of common tendon injuries. Am J Roentgenol. 2009;193:607-618.

Open Access Journal of Sports Medicine

\section{Publish your work in this journal}

Open Access Journal of Sports Medicine is an international, peer-reviewed, open access journal publishing original research, reports, reviews and commentaries on all areas of sports medicine. The manuscript management system is completely online and includes a very quick and fair peer-review system.
Visit http://www.dovepress.com/testimonials.php to read real quotes from published authors. 